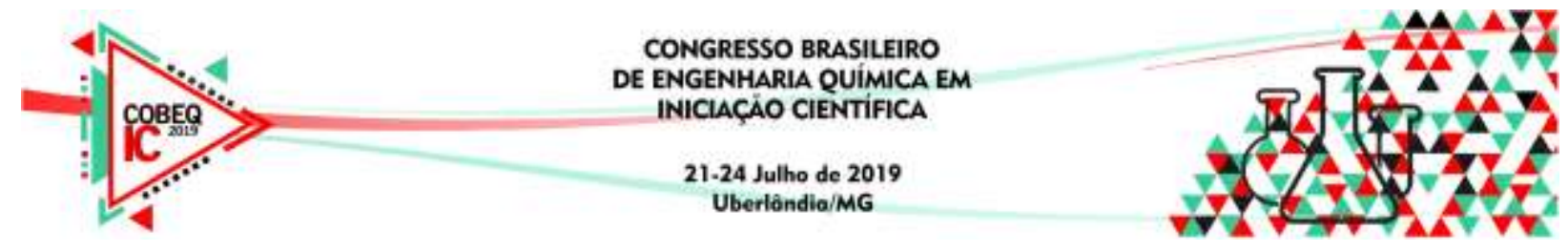

\title{
AVALIAÇÃO DO POTENCIAL DO EMPREGO DE ÁLCOOIS COMO AGENTE ESTABILIZANTE EM MISTURAS BIO- ÓLEO PIROLÍTICO/DIESEL
}

\author{
J. C. MOREIRA ${ }^{1}$, L. M. ASSIS ${ }^{2}$, K. S. T. S. DELASALLES ${ }^{3}$ e W. F. DELASALLES ${ }^{4}$ \\ Universidade Federal do Maranhão, Centro de Ciências Exatas e Tecnologia \\ ${ }^{1,2}$ Coordenação de Engenharia Química, ${ }^{3,4}$ Departamento de Tecnologia Química \\ E-mail para contato: wendellsalles@ @otmail.com
}

\begin{abstract}
RESUMO - O bio-óleo produzido por pirólise rápida ou liquefação da biomassa vem se destacando por ser um produto de grande potencial para atuar como fonte energética. Entretanto, possui algumas propriedades indesejadas quando se considera a sua aplicação como combustível automotivo em motores do ciclo diesel, como por exemplo, alto teor de oxigênio e água, elevada corrosividade e imiscibilidade em diesel. Neste trabalho, foi avaliado o potencial de alguns álcoois como agentes estabilizantes no processo de solubilização de um bio-óleo sintético em diesel através da tecnologia de microemulsificação. Parâmetros que influenciam no processo de solubilização foram avaliados, tais como, razão bioóleo/diesel, natureza e concentração dos álcoois. Os resultados demonstraram que o n-octanol foi o mais eficiente dentre os álcoois estudados para promover a solubilização do sistema. A razão volumétrica bio-óleo/diesel ótima foi 3:7 e a concentração ideal do n-octanol $0,85 \mathrm{M}$. O potencial de aplicação das misturas foi avaliado em termos do seu poder calorífico, massa específica, viscosidade cinemática e índice de viscosidade.
\end{abstract}

\section{INTRODUÇÃO}

Os Combustíveis fósseis são atualmente a fonte energética mais usada mundialmente, contudo, a cada ano, as perspectivas futuras em torno do uso desses recursos não renováveis vêm se tornando cada vez menos atrativas. Em frente a esses direcionamentos, os produtos obtidos a partir de resíduos da biomassa apresentam-se como uma alternativa para a nova abordagem do mercado energético. O bio-óleo, um líquido que pode ser obtido a partir da pirólise da biomassa, vem atraindo muito atenção por ser uma alternativa promissora para substituir os combustíveis fósseis, pois apresenta um grande potencial para o uso em motores de combustão e caldeiras de aquecimento (Liang et al., 2018; Xiu et al., 2012). Entretanto, o seu uso comercial como combustível é muito limitado devido a características como elevada viscosidade, comportamento corrosivo e alto teor de água (Leng et al., 2015). Consequentemente, a sua utilização direta se torna inviável, sendo necessária aplicação de métodos de melhoramento do bio-óleo.

A microemulsificação é uma técnica de melhoramento da qualidade do bio-óleo que consiste na combinação de dois líquidos imiscíveis capazes de formar misturas homogêneas com auxílio de agentes emulsionantes. Através dessa tecnologia, é possível desenvolver 


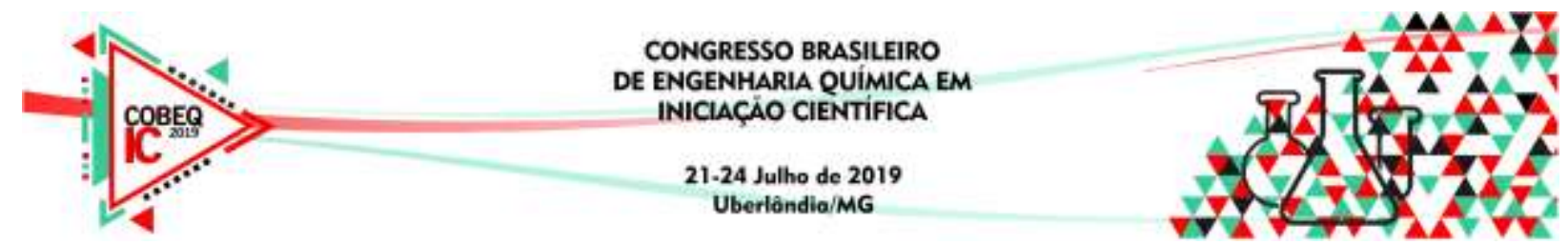

sistemas termodinamicamente estáveis de bio-óleo com diesel ou biodiesel (Leng et al., 2018). Ikura et al. (2003) obtiveram propriedades melhoradas do bio-óleo com teor 5-30\% em diesel quando inserido em microemulsões. No entanto, a utilização dos tensoativos comerciais tem sido um contraponto negativo, devido ao seu elevado custo que dificulta $o$ desenvolvimento em larga escala de processos baseados nesse método.

DeLaSalles et al. (2016) verificaram em seus trabalhos que o aumento da razão cotensoativo/tensoativo levava uma maior solubilização do bio-óleo no sistema, quando do emprego de álcoois de cadeia curta como cotensoativos, evidenciando que a utilização de álcoois de forma isolada poderia ser uma alternativa ao uso dos tensoativos clássicos.

Portanto, o presente trabalho teve como objetivo fundamental avaliar o potencial do emprego de álcoois como agente estabilizante no processo de microemulsificação de bio-óleo em diesel. Diversos parâmetros que afetam a estabilidade da microemulsão foram avaliados, tais como, a razão inicial bio-óleo/diesel (v/v) e a natureza e concentração do álcool. As propriedades da microemulsão combustível também foram analisadas e comparadas com as propriedades do bio-óleo sintético e do diesel.

\section{METODOLOGIA}

\subsection{Material}

A amostra diesel utilizada foi fornecida pelo Laboratório de Análise e Pesquisa em Química Analítica de Petróleo e Bicombustíveis (LAPQAP) da Universidade Federal do Maranhão (UFMA). Glucose, Vanilina, Água Deionizada, Guaiacol, Glioxal, Furfural, Ácido Acético, Metanol e Acetol usados na formulação do bio-óleo e os álcoois n-butanol, nhexanol, n-octanol e isoamílico foram adquiridos junto a Sigma-Aldrich, com pureza superior a 99\%, exceto o glioxal, obtido na forma de solução aquosa a 40\% e o Acetol (90\%).

\subsection{Preparo do Bio-óleo}

O bio-óleo sintético foi formulado com base nos constituintes químicos usados por Wang et al. (2014) com a seguinte composição em massa: $8 \%$ de Glucose, $8 \%$ de Vanilina, $25 \%$ de Água Deionizada, $17 \%$ de Guaiacol, 6\% de Glioxal, 6\% de Furfural, $15 \%$ de Ácido Acético, $7 \%$ de Metanol e $8 \%$ de Acetol. Tal formulação visou a minimizar problemas de reprodutibilidade dos dados da pesquisa uma vez que o bio-óleo real é relativamente instável quimicamente. Incialmente, adicionou-se a glucose, vanilina e água deionizada e agitou-se por cerca de 10 minutos até completa solubilização, em seguida, os demais componentes foram inseridos. A mistura resultante foi continuamente agitada por cerca de duas horas.

\subsection{Preparo das Misturas}

Com um volume total de $10 \mathrm{~mL}$ (variando-se a razão inicial bio-óleo/diesel e a natureza do cotensoativo), massas de bio-óleo, diesel e álcool foram pesadas e misturadas em erlenmeyer sob agitação magnética por $15 \mathrm{~min}$. Posteriormente, as amostras foram transferidas para cones graduados e deixadas em repouso durante $24 \mathrm{~h}$, onde se observava uma separação de fases, sendo a fase superior a fase microemulsionada e a fase inferior bio- 


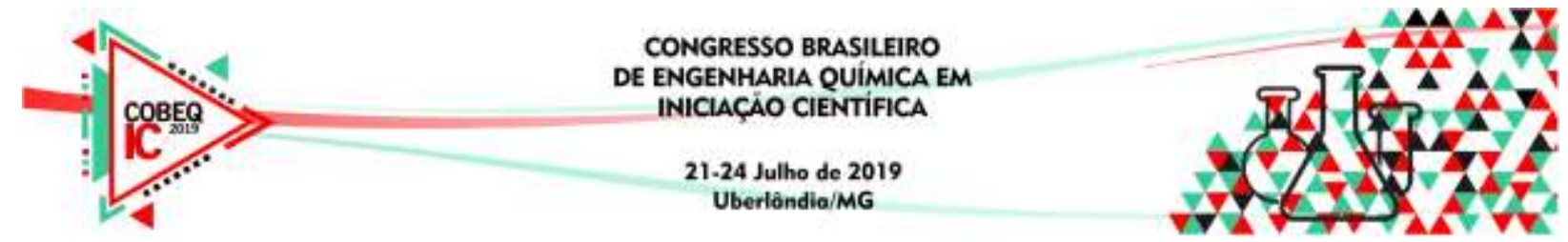

óleo residual. Todos os ensaios foram realizados a temperatura ambiente $\left(25^{\circ} \mathrm{C}\right)$. A eficiência do sistema no processo de solubilização de bio-óleo foi avaliada em termos de dois parâmetros de otimização, definidos como parâmetro $S$ (volume de bio-óleo solubilizado por volume de diesel) e parâmetro E (volume de bio-óleo solubilizado por mol de cotensoativo), conforme o trabalho de Wang et al. (2014).

\section{4. Caracterização}

A caracterização da microemulsão, bio-óleo e do diesel foram realizadas no Laboratório de análises em Química Analítica de Petróleo e Biocombustíveis da Universidade Federal do Maranhão por meio de análises de massa específica (Density Meter DMA $4500 \mathrm{M}$ ), poder calorífico (IKA C2000), viscosidade (HVB 438 visco bath). A estabilidade da microemulsão foi avaliada em termos da determinação do índice de viscosidade, conforme trabalho de Chiaramonti et al. (2003).

O índice de viscosidade é calculado

\section{RESULTADOS E DISCUSSÃO}

\subsection{Efeito da Natureza do Álcool e da Razão Bio-óleo/Diesel}

Os resultados das análises da razão inicial B/D (v/v) e da influência dos diferentes tipos de álcoois na solubilização de bio-óleo estão dispostos na Figura 1. As concentrações dos álcoois estudados foram fixadas em $0,75 \mathrm{M}$.

Figura 1 - Influência da natureza do álcool e da razão B/D de solubilização do bio-óleo em diesel.

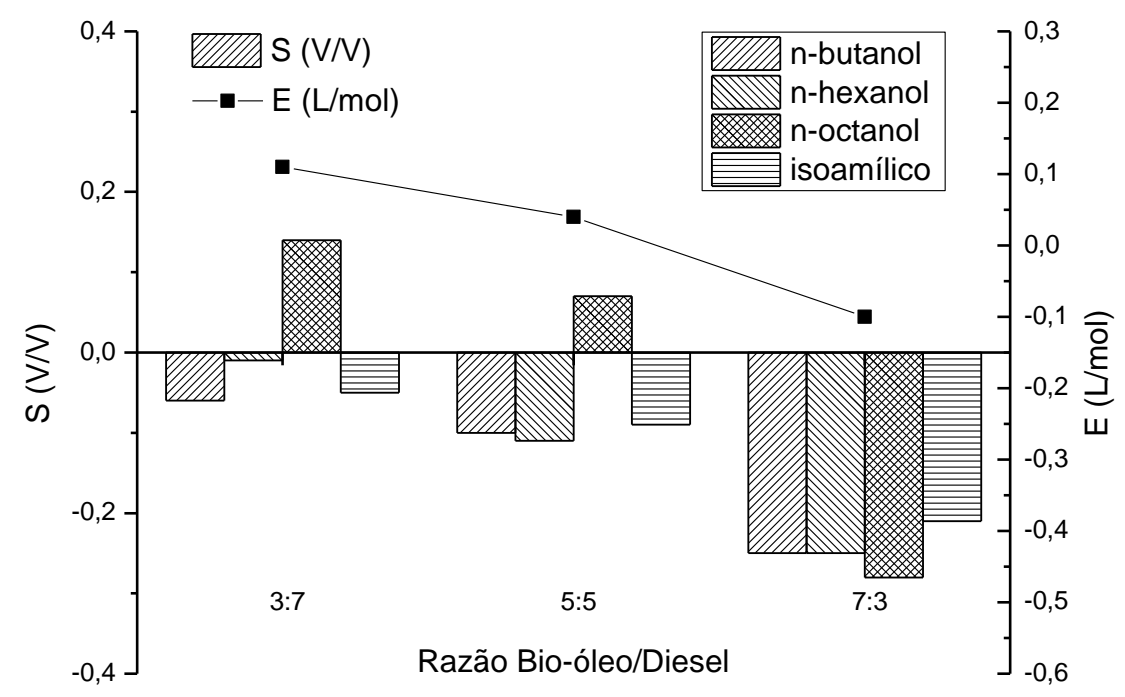

Ao analisar a atuação dos álcoois como estabilizante, percebe-se que a maior parte deles não promoveu melhoria na solubilização do bio-óleo em diesel, apresentando um incremento negativo, ou seja, ao invés de se verificar uma redução no volume de bio-óleo residual devido a uma solubilização parcial deste na fase diesel observou-se um aumento no volume de bio- 


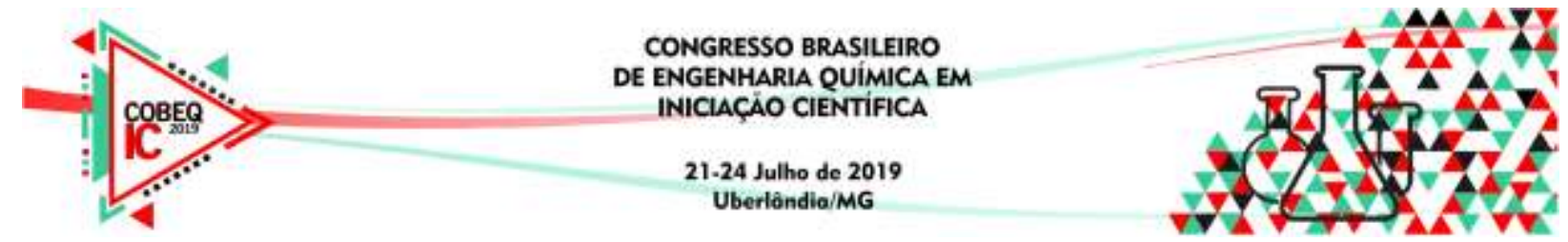

óleo em função do álcool adicionado ao sistema se solubilizar nesta fase. Dentre os álcoois avaliados, somente o n-octanol foi capaz de promover uma solubilização parcial de bio-óleo na fase diesel, obtendo valores de parâmetro $S$ (volume bio-óleo solubilizado por volume de diesel) em torno de 0,14 na razão bio-óleo/diesel (v/v) de 3:7. Avaliando-se o volume de bioóleo solubilizado por mol de álcool empregado, representado pelo parâmetro E, nota-se que o n-octanol obteve maior eficiência na razão 3:7 tendo um valor de $E_{\text {máx }}=0,13$. Observa-se também uma tendência do decaimento da eficiência do álcool com o aumento da razão B/D.

Os resultados obtidos revelam a capacidade de álcoois (especificamente o n-octanol em nosso caso) de atuar como agente tensoativo, conforme relatado nos trabalhos de DeLuna et al. (2017). A superfície ativa desses componentes proporciona a redução da tensão superficial entre fases imiscíveis, como no caso da mistura bio-óleo/diesel, podendo levar o sistema à formação de uma emulsão ou microemulsão. De modo semelhantemente aos resultados expostos neste trabalho, Wang et al. (2014) avaliando o efeito do n-octanol, n-heptanol, nhexanol, n-pentanol e n-butanol como cotensoativos concluíram que o n-octanol era um coagente ideal de solubilização de bio-óleo em diesel, no entanto, nestes trabalhos o octanol atuava de fato como um cotensoativo associado a um tensoativo (Span80).

\subsection{Efeito da Concentração do Álcool}

A influência da concentração do álcool no processo de solubilização do bio-óleo é apresentada na Figura 2. Para a análise, foi tomado como base as condições pré-estabelecidas com razão B/D inicial de 3:7 em volume e n-octanol como álcool. Variou-se a concentração do álcool de 0,75 a $1,15 \mathrm{M}$.

Figura 2 - Efeito da concentração do n-octanol na solubilização do bio-óleo em diesel.

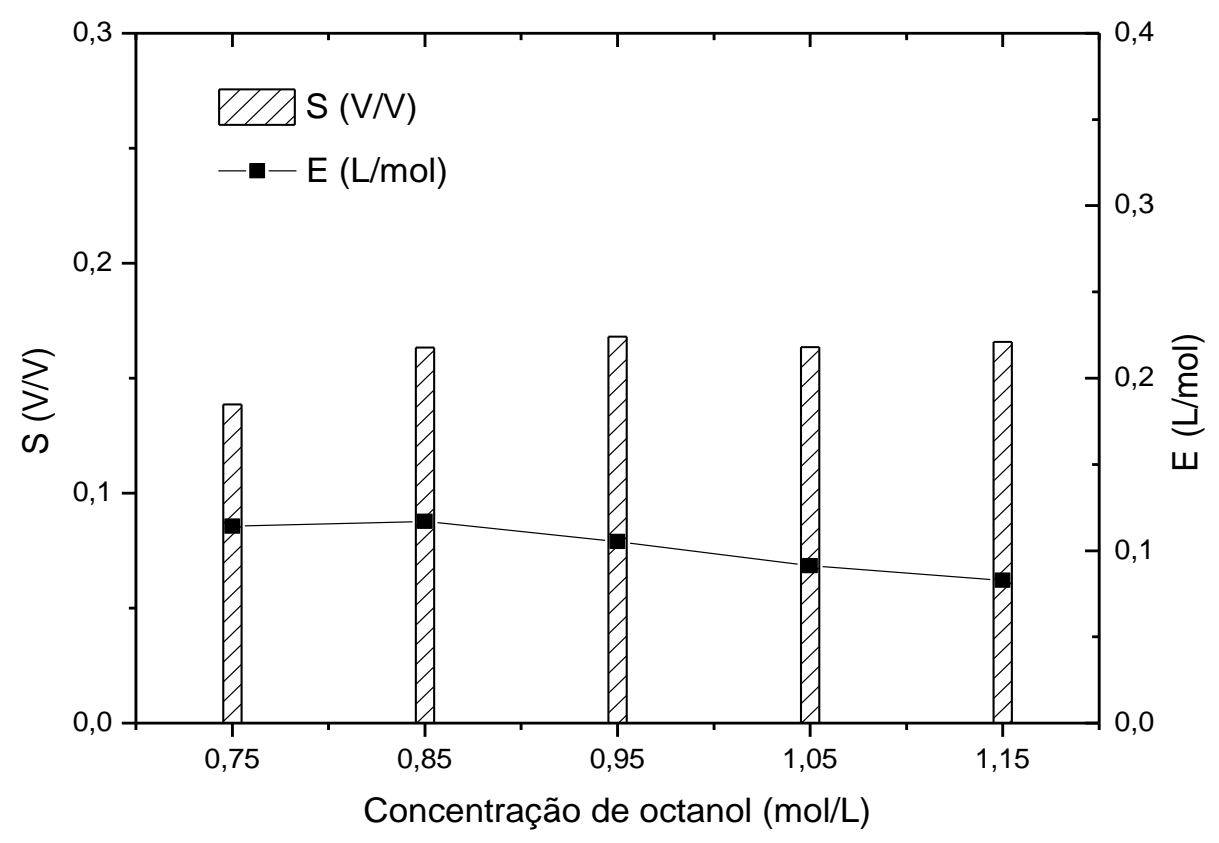




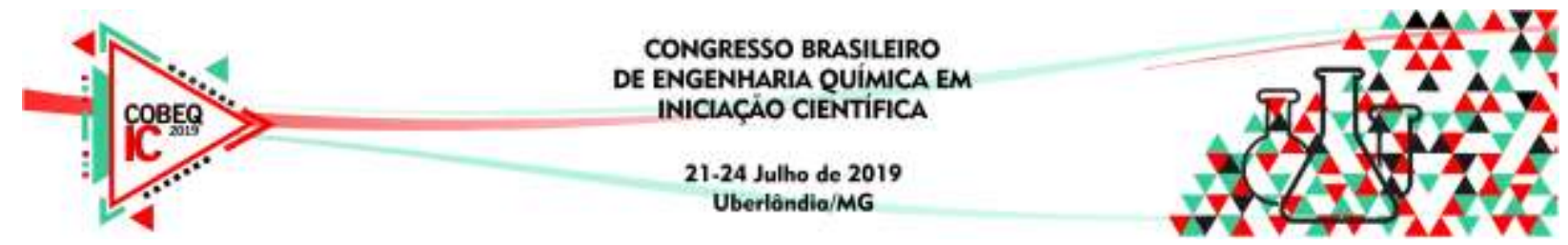

Ao se passar de $0,75 \mathrm{M}$ para $0,85 \mathrm{M}$ observa-se um leve aumento no teor de bio-óleo solubilizado, no entanto, a partir desta concentração o teor de bio-óleo solubilizado praticamente não sofreu alteração, o que evidencia a existência de um limite máximo na capacidade do n-octanol com um agente de solubilização do bio-óleo na mistura. No que diz respeito a eficiência do álcool no processo (avaliado pelo parâmetro E), também se atinge um máximo na concentração de $0,85 \mathrm{M}$.

De acordo com Jiang e Ellis (2009), existe uma concentração ótima de octanol para produzir misturas bio-óleo/diesel estáveis. Quando as concentrações são muito elevadas pode ocorrer a desestabilização por coalescência, em contrapartida, em baixas concentrações o sistema está sujeito a aglomerações de gotículas de óleo. Logo, é importante a definição de uma concentração ideal para favorecer a máxima eficiência de solubilização.

\subsection{Caracterização}

A caracterização foi realizada tanto para a microemulsão selecionada (fase microemulsionada obtida empregando-se octanol na concentração de 0,85 M como agente estabilizante e razão (v/v) bio-óleo/diesel de 3:7), quanto para o bio-óleo e o diesel. Os resultados das análises de massa específica, poder calorífico, viscosidade e índice de viscosidade são apresentados na Tabela 1.

Tabela 1 - Caracterização das amostras de diesel, bio-óleo e microemulsão

\begin{tabular}{ccccc}
\hline & PCS $(\mathrm{MJ} / \mathrm{kg})$ & $\rho\left(\mathrm{g} / \mathrm{cm}^{3}\right)$ & $v\left(\mathrm{~mm}^{2} / \mathrm{s}\right)$ & $\mathrm{IV}$ \\
\hline Bio-óleo & 18,27 & 1,116 & 2,320 & 0,162 \\
Diesel & 44,86 & 0,853 & 3,770 & 0,008 \\
Microemulsão & 40,08 & 0,879 & 4,038 & 0,047 \\
\hline
\end{tabular}

Observa-se que os valores das massas específicas das microemulsões situaram-se levemente acima dos valores obtidos para o diesel puro, influenciadas diretamente pela presença do bio-óleo nesta fase. Os valores das viscosidades cinemáticas das microemulsões também foram superiores aos valores obtidos para o diesel puro, fato que se justifica pela presença do octanol na composição das microemulsões. Como era esperado, o poder calorífico das microemulsões se apresentou inferior ao poder calorífico do diesel, no entanto, bem superior ao poder calorífico do bio-óleo puro. É importante ressaltar que o poder calorífico da microemulsão está diretamente relacionado ao volume de bio-óleo solubilizado, de modo que pode ser elevado, ou diminuído, alterando-se o volume de bio-óleo solubilizado. Em relação ao índice de viscosidade, sabe-se que quanto menor o seu valor, mais estável é a mistura, de modo que o valor obtido indica que a microemulsão avaliada possui boa estabilidade.

\section{CONCLUSÃO}

Os resultados apresentados confirmam o potencial do emprego da tecnologia de microemulsões em processos de melhoramento do bio-óleo empregando-se álcoois como agente de solubilização, em contrapartida ao uso dos tensoativos clássicos. Foram obtidas misturas estáveis bio-óleo/diesel na razão de $0,17 \mathrm{~mL}$ de bio-óleo por $\mathrm{mL}$ de diesel com 


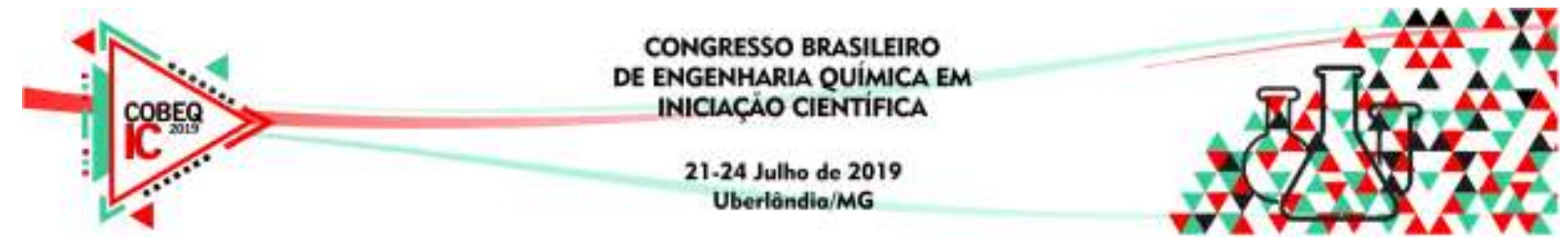

propriedades combustíveis semelhantes às propriedades do diesel, sinalizando que tal procedimento tem potencial real de aplicação no aproveitamento do bio-óleo como combustível automotivo. No mais, as propriedades das microemulsões obtidas ainda podem ser otimizadas, visto que as condições "ótimas" para o sistema microemulsionado foram definidas apenas em termos de parâmetros de otimização baseados exclusivamente na eficiência no tensoativo e no volume de bio-óleo solubilizado, sem uma preocupação mais efetiva com o impacto destes parâmetros nas propriedades combustíveis da microemulsão.

\section{REFERÊNCIAS}

CHIARAMONTI, D.; BONINI, M., FRATINI, E., TONDI, G., GARTNEr, K., BRIDGWATER, A. V., GRIMM, H. P., SOLDAINI, I., WEBSTER, A., BAGLIONI, P., Development of emulsions from biomass pyrolysis liquid and diesel and their use engines - part 1: emulsion production, Biomass and Bioenergy, 25, 2003, 85-99.

DE LUNA, M. D. G.; CRUZ, L. A. D.; CHEN, W. H.; LIN, B. J.; HSIEH, T. H. Improving the stability of diesel emulsions with high pyrolysis bio-oil content by alcohol cosurfactants and high shear mixing strategies. Energy, v. 141, p. 1416-1428, 2017.

FERREIRA DE LA SALLES, W.; TEIXEIRA DA SILVA DE LA SALLES, K.; FREIRE, E. A. R.; ASSIS, L. M. Estudo da capacidade de solubilização de bio-óleo em diesel por meio da tecnologia de microemulsões empregando brij-93 como tensoativo. In: XXI Congresso Brasileiro de Engenharia Química, 2016

IKURA, M.; STANCIULESCU, M.; HOGAN, E. Emulsification of pyrolysis derived bio-oil in diesel fuel. Biomass. Bioenerg., v. 24, n. 3, p. 221-232, 2003.

JIANG, X.; ELLIS, N. Upgrading bio-oil through emulsification with biodiesel: mixture production. Energ. Fuel, v. 24, n. 2, p. 1358-1364, 2009.

LENG, L.; Li, H.; YUAN, X.; ZHOU, W.; HUANG, H. Bio-oil upgrading by emulsification/microemulsification: A review. Energy, 2018

LIANG, J.; QIAN, Y.; YUAN, X.; LENG, L.; ZENG, G.; JIANG, L.; SHAO, J.; LUO, Y.; DING, X.; YANG, Z.; LI, X. Span80/Tween80 stabilized bio-oil-in-diesel microemulsion: Formation and combustion. Renew. Energy., v. 126, p. 774-782, 2018.

LENG, L.; YUAN, X.; CHEN, X.; HUANG, H.; WANG, H.; LI, H.; ZHU, R.; Li, S.; ZENG, G. Characterization of liquefaction bio-oil from sewage sludge and its solubilization in diesel microemulsion. Energy, v. 82, p. 218-228, 2015.

WANG, X. L.; YUAN, X. Z.; HUANG, H. J.; LENG, L. J.; LI, H.; PENG, X.; WANG, H.; LIU, Y.; ZENG, G. M. Study on the solubilization capacity of bio-oil in diesel by microemulsion technology with Span80 as surfarctant. Fuel Process. Technol., v. 118, 2014.

XIU, S.; SHAHBAZI, A. Bio-oil production and upgrading research: A review. Renew. Sust Energy. Rev., v. 16, n. 7, p. 4406-4414, 2012. 